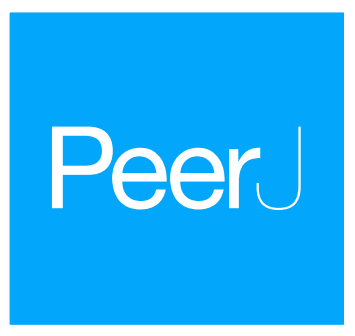

\title{
The complete chloroplast genome of Fagus crenata (subgenus Fagus) and comparison with F. engleriana (subgenus Engleriana)
}

\author{
James R. P. Worth ${ }^{1}$, Luxian $\mathrm{Liu}^{2}$, Fu-Jin $\mathrm{Wei}^{1}$ and Nobuhiro Tomaru ${ }^{3}$ \\ ${ }^{1}$ Department of Forest Molecular Genetics and Biotechnology, Forestry and Forest Products \\ Research Institute, Tsukuba, Japan \\ ${ }^{2}$ Key Laboratory of Plant Stress Biology, Laboratory of Plant Germplasm and Genetic \\ Engineering, College of Life Sciences, Henan University, Kaifeng, China \\ ${ }^{3}$ Graduate School of Bioagricultural Sciences, Nagoya University, Nagoya, Japan
}

\section{ABSTRACT}

This study reports the whole chloroplast genome of Fagus crenata (subgenus Fagus), a foundation tree species of Japanese temperate forests. The genome has a total of $158,227 \mathrm{bp}$ containing 111 genes, including 76 protein-coding genes, 31 tRNA genes and 4 ribosomal RNA genes. Comparison with the only other published Fagus chloroplast genome, F. engeleriana (subgenus Engleriana) shows that the genomes are relatively conserved with no inversions or rearrangements observed while the proportion of nucleotide sites differing between the two species was equal to 0.0018 . The six most variable regions were, in increasing order of variability, $p s b \mathrm{~K}-p s b \mathrm{I}$, trn G-psbfM, rpl32, trn V, $n d h \mathrm{I}-n d h$ and $n d h D-p s a \mathrm{C}$. These highly variable chloroplast regions in addition to 160 chloroplast microsatellites identified (of which 46 were variable between the two species) will provide useful genetic resources for studies of the inter- and intra-specific genetic structure and diversity of this important northern hemisphere tree genus.

Submitted 9 January 2019

Accepted 24 April 2019

Published 7 June 2019

Corresponding author

James R. P. Worth,

jrpw2326@affrc.go.jp

Academic editor

Axel Tiessen

Additional Information and Declarations can be found on page 8

DOI 10.7717/peerj.7026

(c) Copyright

2019 Worth et al.

Distributed under

Creative Commons CC-BY 4.0

\section{OPEN ACCESS}

Subjects Biodiversity, Evolutionary Studies, Genetics, Plant Science

Keywords Beech, Chloroplast SSRs, Fagaceae phylogeny, Fagus crenata, Whole chloroplast genome, Chloroplast microsatellites

\section{INTRODUCTION}

The genus Fagus is a major tree of temperate forests of the northern hemisphere with two informal subgenera recognized (Shen, 1992): Engleriana with three species and Fagus with seven species (Oh, 2015; Renner et al., 2016). The genus has been the focus of intensive genetic studies over the last 30 years enabling insights into relationships of the extant species (Denk, Grimm \& Hemleben, 2005), the impact of the interglacial-glacial cycles on extant genetic diversity (Fujii et al., 2002; Magri et al., 2006) and predictions of the impacts of ongoing climate change (Csilléry et al., 2014). However, despite the significance of the genus there remains a dearth of Next Generation Sequencing based-genetic resources for Fagus, including for the chloroplast genome, with the whole chloroplast genome of only a single species, the Chinese endemic F. engleriana of subgenus Engleriana (Yang et al., 2018), so far published. 
This study reports the whole chloroplast genome of the Japanese endemic Fagus crenata, the first reported of subgenus Fagus. This species is a foundation tree of Japan's cool temperate forest ecosystem and is distributed widely from the mountains of southern Kyushu $\left(31.4^{\circ} \mathrm{N} 130.8^{\circ} \mathrm{E}\right)$ to southern Hokkaido $\left(42.8^{\circ} \mathrm{N} 140.2^{\circ} \mathrm{E}\right)$. Phylogeographic studies based on Sanger sequencing of small portions of the chloroplast genome have revealed strong geographic structuring of chloroplast haplotypes (Fujii et al., 2002; Okaura \& Harada, 2002) that, combined with fossil pollen data (Tsukada, 1982), suggests the species persisted in multiple coastal refugia and has occupied most of its current wide geographic range in the postglacial. Here we report the whole chloroplast genome sequence of $F$. crenata and compare it to the genome of F. engleriana (subgenus Engleriana). This data will be a useful genetic resource for investigating the phylogenetic relationship of Fagus and for developing chloroplast-based genetic markers, including both single nucleotide polymorphism- and microsatellite-based markers.

\section{MATERIALS AND METHODS}

\section{Next Generation Sequencing and chloroplast genome assembly}

Whole genomic DNA was extracted from a single sample of $F$. crenata collected from Daisengen Peak, Hokkaido, Japan $\left(41.616^{\circ} \mathrm{N}-140.1333^{\circ} \mathrm{E}\right)$ representing the F. crenata chloroplast haplotype A (following Fujii et al., 2002) using a modified CTAB protocol (Doyle, 1990). DNA concentration and quality were assessed by agarose gel electrophoresis and a Qubit 2.0 fluorometer (Life Technologies). A total of $9 \mu \mathrm{g}$ of DNA was sent to the Beijing Genomic Institute where short-size Truseq DNA libraries were constructed and paired-end sequencing $(2 \times 100 \mathrm{bp})$ was performed on an Illumina HiSeq2000 Genome Analyser resulting in a total of 7,223,910 reads (the raw sequence reads are deposited in NCBI BioProject Database Accession number: PRJNA528838).

Assembly of chloroplast DNA from the whole genomic sequencing data was undertaken in Novoplasty 2.6.3 (Dierckxsens, Mardulyn \& Smits, 2016), a seed-and-extend algorithm that is designed for the specific purpose of assembling chloroplast genomes from whole genome sequencing data, starting from a chloroplast seed sequence (trnK-matK of haplotype A: Genbank accession AB046492). This resulted in nine chloroplast contigs varying in length from 2,748 to 43,982 bp constructed from 230,360 chloroplast reads (3.19\% of the total reads) with an average read coverage of the chloroplast genome of 145 . The nine contigs were ordered and oriented using the $F$. engleriana whole chloroplast genome (KX852398) as a reference and the complete chloroplast sequence of $F$. crenata was constructed by connecting overlapping terminal sequences. Sanger sequencing was undertaken to check the accuracy of assembly of the joins of the nine contigs and the inverted repeat and single copy regions and also the sequences of the most diverged sites between F. crenata and F. engleriana (see "Results and Discussion"). A total of 8,146 bp was sequenced using 15 primer pairs and no differences were observed with the F. crenata genome apart from those due to inaccurate sequence at the terminal ends of the Sanger sequences. 


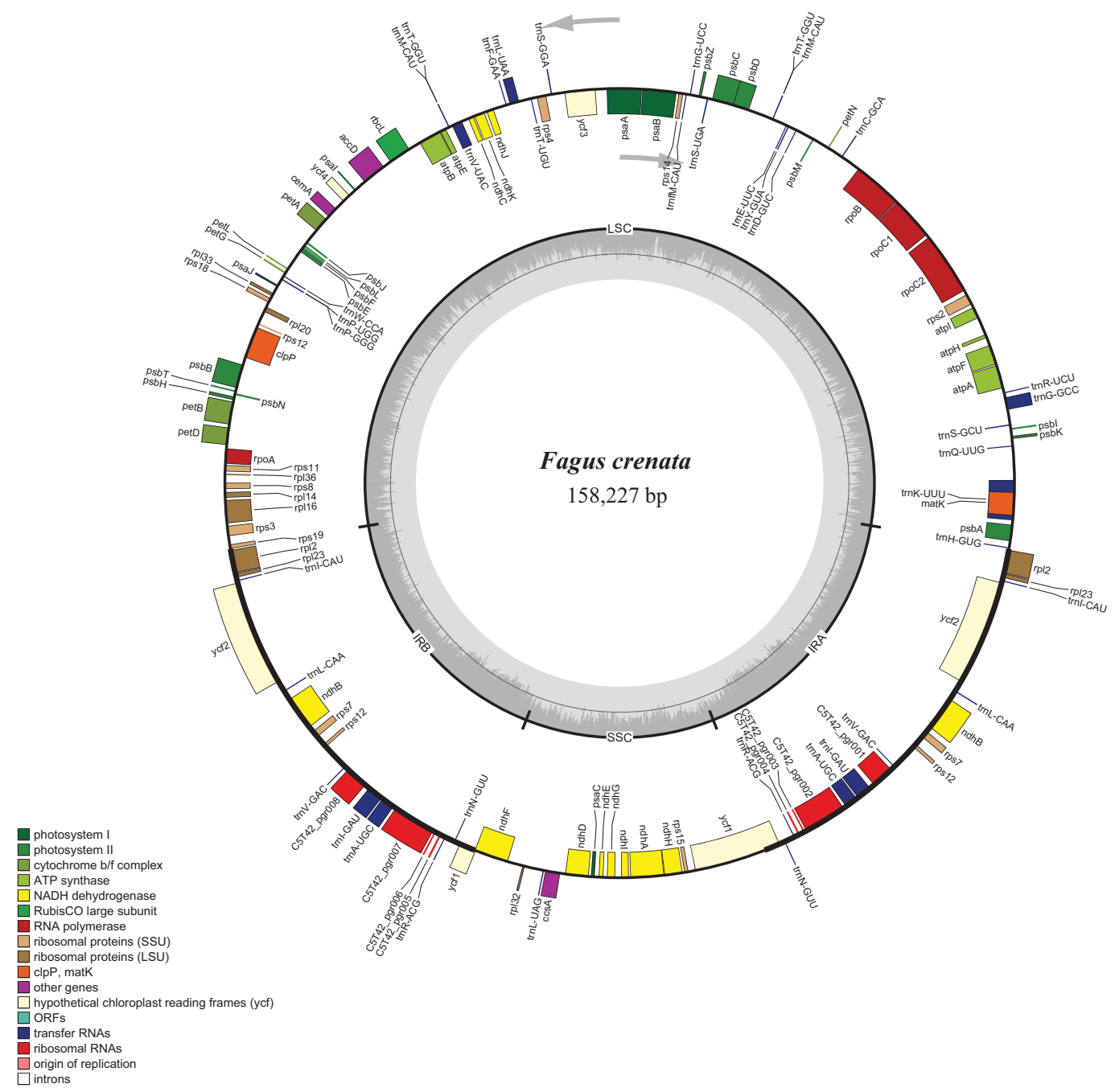

Figure 1 Chloroplast genome map of Fagus crenata. Genes inside the circle are transcribed clockwise, genes outside are transcribed counter-clockwise. The light gray inner circle corresponds to the AT content, the dark gray to the GC content. Genes belonging to different functional groups are shown in different colors. The position of the large single copy (LSC) region, small single copy (SSC) region and two inverted repeats (IRa and IRb) are also indicated. Full-size $\underset{0}{ }$ DOI: 10.7717 /peerj.7026/fig-1

\section{Chloroplast genome annotation}

The annotation of the chloroplast genome was performed using the online program Dual Organellar Genome Annotator (Wyman, Jansen \& Boore, 2004). Initial annotation, putative starts, stops and intron positions were determined according to comparisons with homologous genes of F. engleriana chloroplast genome using Geneious v9.0.5 (Biomatters, Auckland, New Zealand). A circular gene map was drawn by the OrganellaGenomeDRAW tool (OGDRAW) followed by manual modification (Lohse, Drechsel \& Bock, 2007).

\section{Phylogenetic analysis and assessment of divergent regions}

A multiple sequence alignment of $F$. crenata, F. engleriana, representative whole chloroplast genomes of the Fagaceae family and outgroups from Betulaceae, Juglandaceae and 


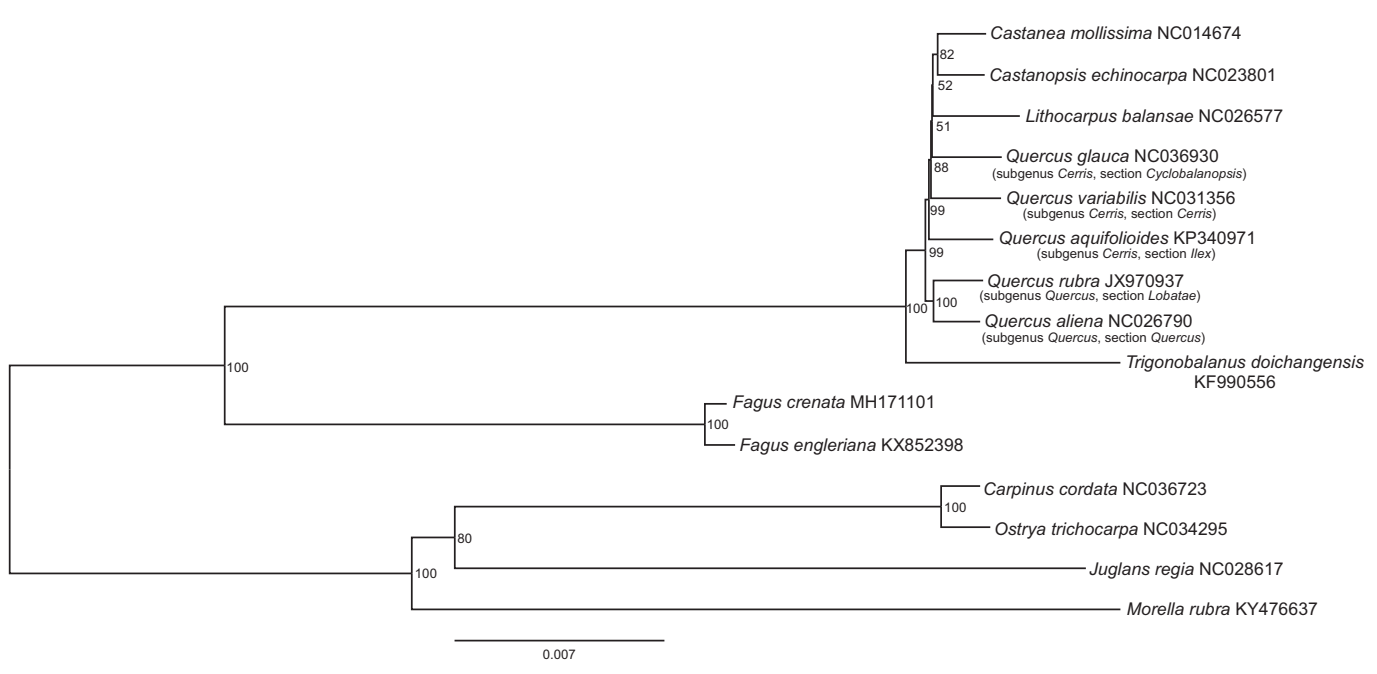

Figure 2 The best scoring maximum likelihood phylogenetic tree based on 143,882 bp of nongapped sequence in a Gblock alignment of the whole chloroplast genomes of Fagus crenata, $F$. engleriana, representative genera of the Fagaceae and outgroups from Betulaceae, Juglandaceae and Myricaceae. A total of $11.48 \%$ of the sites were variable. The Genbank accession number of each chloroplast genome is shown after the species name. Full-size $\boldsymbol{\oplus}$ DOI: 10.7717 /peerj.7026/fig-2

Myricaceae obtained from Genbank was constructed using T-Coffee using default parameters (Notredame, Higgins \& Heringa, 2000). Subsequently, Gblocks v0.91b (Castresana, 2000) was used to identify homologous blocks of DNA and remove poorly aligned and divergent regions of the chloroplast genomes. RAxML NG (Kozlov et al., 2018) was then used to construct a maximum likelihood phylogenetic tree using the most appropriate DNA substitution model, TVM+I+G, as estimated in jModelTest 2.1.10 (Darriba et al., 2012) and 1,000 bootstrap replicates.

Pairwise nucleotide differences (p-distance) between the sequences of the Gblocks alignment were calculated in Mega 7 (Kumar, Stecher \& Tamura, 2016) excluding parts of the sequence alignment with gaps. The coding genes, non-coding regions and intron regions were compared between the alignment of the two Fagus chloroplast genomes to detect divergence hotspots. We examined 101 regions (39 coding genes, 52 intergenic spacers and 10 intron regions) of the two Fagus species for nucleotide variability ( $\mathrm{Pi}$ ) values calculated in DnaSP v5.0 (Librado \& Rozas, 2009).

\section{Identification of chloroplast microsatellites}

Chloroplast microsatellite regions shared in both $F$. crenata and $F$. engleriana were searched for in an alignment of the two full chloroplast genomes (constructed by MAFFT v7.308 (Katoh et al., 2002) under default settings) using Phobos Tandem Repeat Finder (Mayer, 2008) implemented in Geneious v9.0.5. Microsatellite in either of the sequences with a repeat unit length of 1-2 bp were searched for using a minimum length of $10 \mathrm{bp}$ while those with a repeat length of 3-6 bp were selected if they displayed three or more repeats.

\section{RESULTS AND DISCUSSION}

The assembled whole chloroplast genome of $F$. crenata has a total of 158,227 bp (Fig. 1: Genbank accession number MH171101) and consisted of an 87,557 bp large single copy 


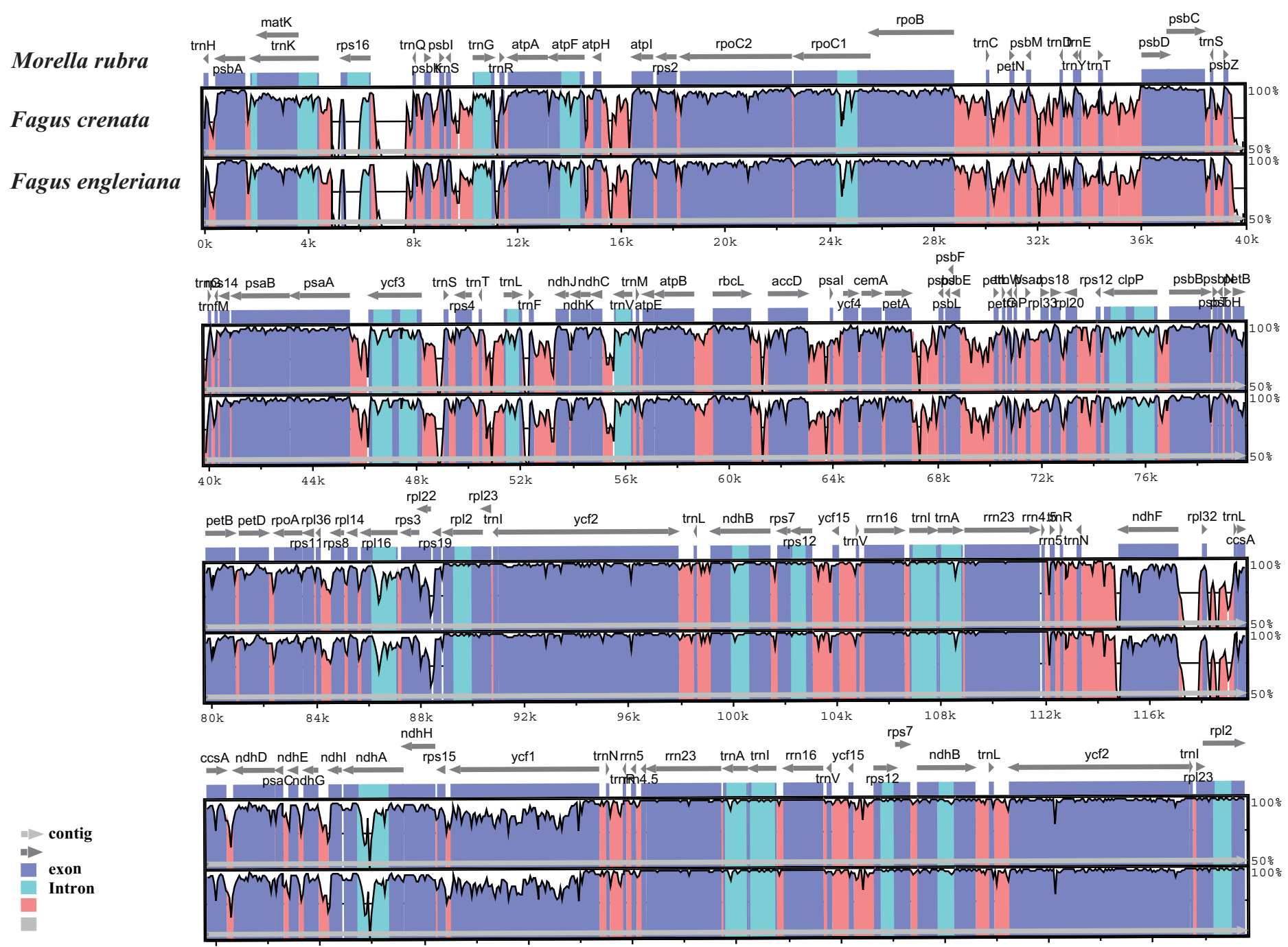

Figure 3 Visualization of alignment of the two Fagus chloroplast genome sequences, with Morella rubra (Myricaceae, Fagales) as a reference. The horizontal axis indicates the coordinates within the chloroplast genome. The vertical scale indicates the percentage of identity, ranging from 50 to $100 \%$. Genome regions are color coded as protein coding, intron, mRNA and conserved non-coding sequence (CNS).

Full-size

region, a 18,928 bp small single copy region and two inverted repeats $25,871 \mathrm{bp}$ in length. The genome contained 111 genes, including 76 protein-coding genes, 31 tRNA genes and 4 ribosomal RNA genes (see DatasetS1 for the genbank file of the chloroplast genome). The Gblocks alignment consisted of 143,882 bp of non-gapped sequence of which $11.48 \%$ of sites were variable (see DatasetS2 for Gblocks alignment). The resulting best ML tree had similar relationships to previous studies with Fagus as sister to all other Fagaceae (Manos \& Steele, 1997) (Fig. 2). Fagus crenata and F. engleriana formed a strongly diverged clade consistent with previous evidence of the large divergence of Fagus from all other Fagaceae genera (Heenan \& Smissen, 2013). The proportion of nucleotide sites that differed (p-distance) between F. crenata and F. engleriana was 0.0018 which was lower than any other pairwise differences observed including between five Quercus species which 


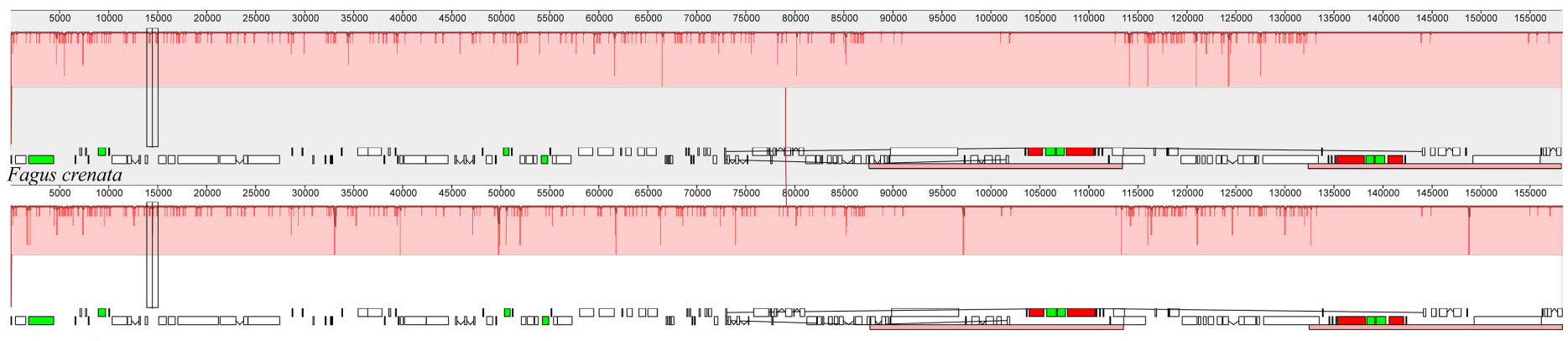

Fagus engleriana

Figure 4 A MAUVE (Darling et al., 2004) alignment of Fagus crenata and F. engleriana chloroplast genomes showing the lack of rearrangements between the chloroplast genomes of the two species. The Fagus crenata genome is shown at top as the reference. Within each of the alignment, local collinear blocks are represented by blocks of the same color connected by lines.

Full-size D DOI: 10.7717/peerj.7026/fig-4

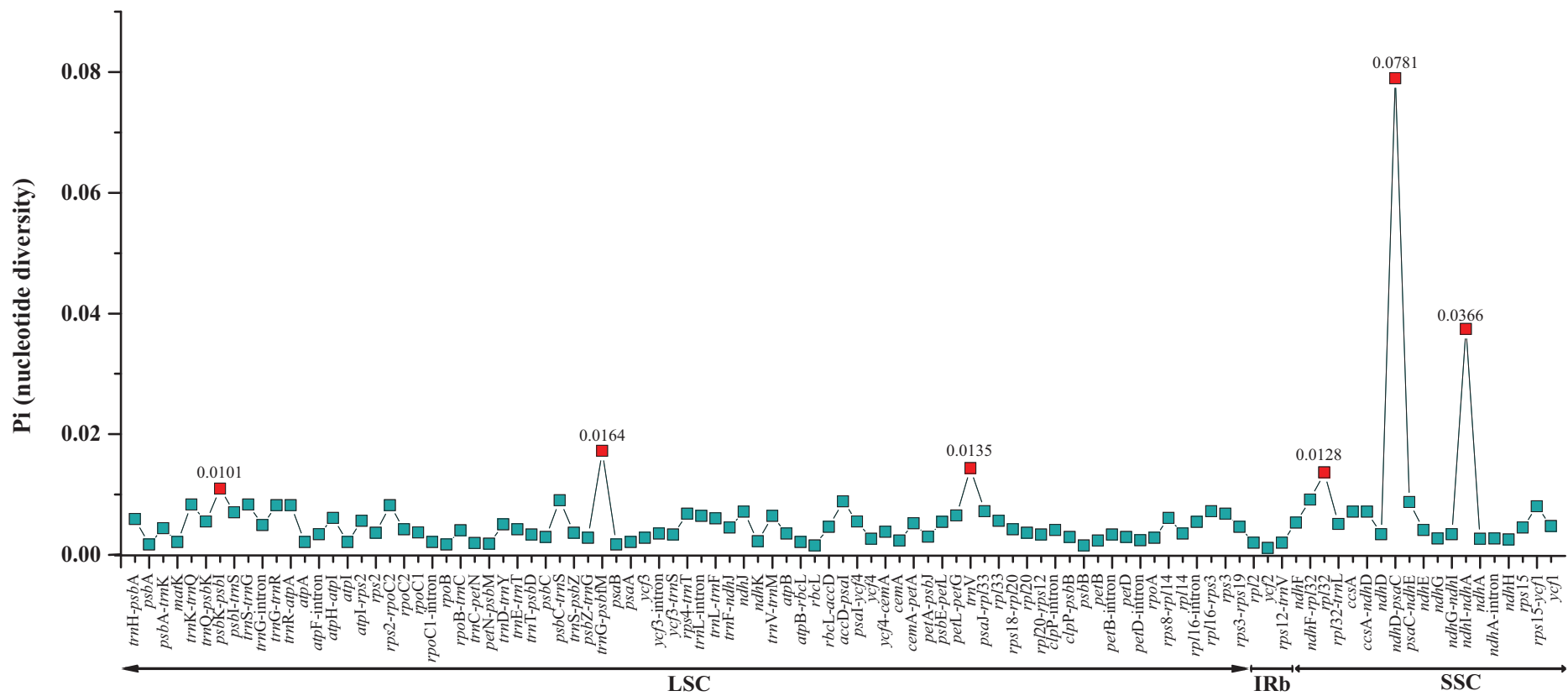

Figure 5 Comparative analysis of the nucleotide diversity $(\mathbf{P i})$ values between the 101 regions ( 39 coding genes, 52 intergenic spacers, and 10 intron regions) of the whole chloroplast genomes of the Fagus crenata and $\boldsymbol{F}$. engleriana. The regions are ordered based on their position in the chloroplast genomes. Full-size 0 DOI: 10.7717 /peerj.7026/fig-5

had values between 0.0035 and 0.0047 (average $=0.0042$ ) (see DatasetS3 for a matrix of p-distances).

The two Fagus chloroplast genomes were relatively conserved (Fig. 3) with the IR region more conserved than both the large single copy (LSC) and small single copy (SSC) regions. We did not detect either inversions or translocations among the two genome sequences, and no rearrangement occurred in gene organization after verification (Fig. 4). There was high variation in nucleotide diversity values observed between the 101 regions of the two Fagus species with values ranging from 0.0003 ( $y c f 2$ gene) to 0.0781 ( $n d h \mathrm{D}-p s a \mathrm{C}$ ) (Fig. 5). The six most variable regions were, in increasing order of variability, $p s b \mathrm{~K}-p s b \mathrm{I}, t r n \mathrm{G}-p s b \mathrm{fM}, r p l 32, \operatorname{trn\mathrm {V}}, n d h \mathrm{I}-n d h$ and $n d h D-p s a \mathrm{C}$ of which four are located in 


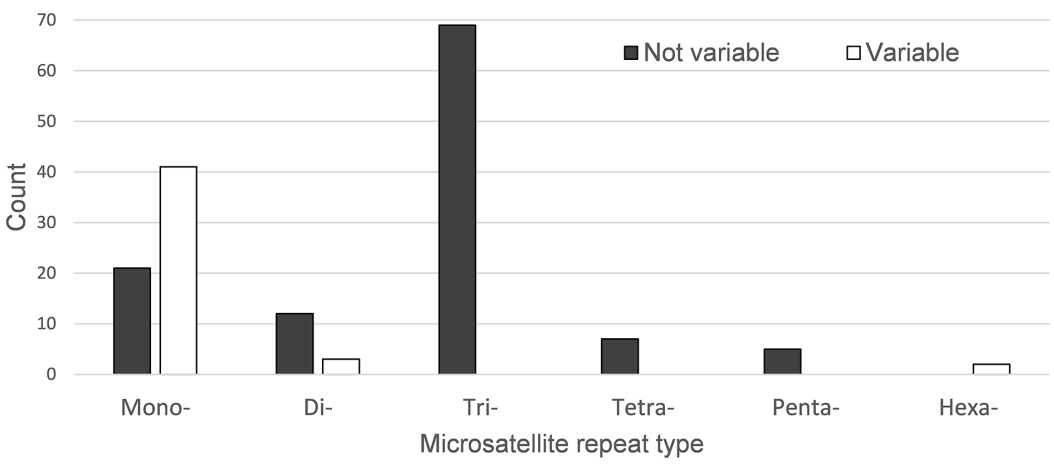

Figure 6 The number of chloroplast microsatellites of each repeat type shared in Fagus crenata and F. engleriana. Microsatellites that displayed no size variation between the two species are indicated by black bars while those that did are indicated by white bars. Note that the number of variable tri-, tetraand penta-nucleotide chloroplast microsatellites was zero. Full-size $\square$ DOI: 10.7717/peerj.7026/fig-6

(a)

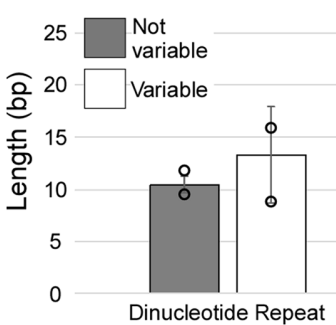

Fagus engleriana
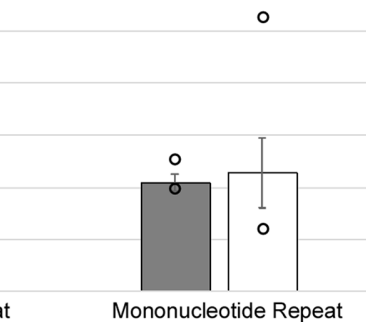

(b)

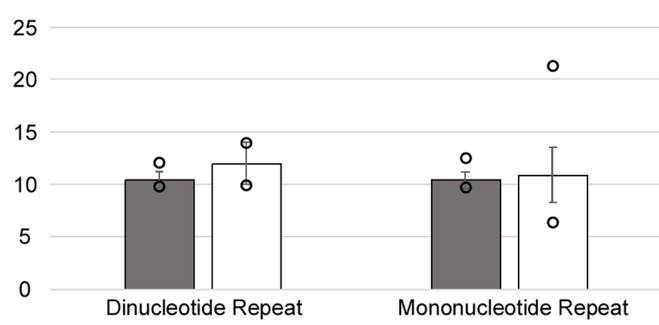

Figure 7 The average length (bp) of variable versus non-variable chloroplast microsatellites for both mono- and di-nucleotide repeat motif types observed in both (A) Fagus crenata and (B) F. engleriana including the standard deviation (error bars) and minimum and maximum lengths (empty circles).

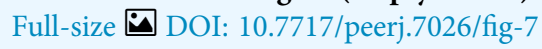

the LSC region and two in the SSC region (Fig. 5). The nucleotide diversities of these variable regions between $F$. crenata and $F$. engleriana were higher than observed within some other studies of Fagaceae genera including East Asian (Yan et al., 2018) and Mediterranean oaks (Vitelli et al., 2017).

A total of 160 chloroplast microsatellites with a repeat unit length between 1 and 6 bp were identified based on the selection criteria in the two species of which mono- and tri-nucleotide repeat microsatellites were the most abundant with a frequency of $38.7 \%$ and $43.1 \%$, respectively. This abundance of mono- and tri-repeats in the chloroplast is similar to a range of other angiosperms (Melotto-Passarin et al., 2011). Of these microsatellites, 46 displayed size variation between $F$. crenata and $F$. engleriana (see DatasetS4 for a table with details of all 46 variable chloroplast microsatellites). The majority $(66.1 \%)$ of the variable chloroplast microsatellites were mono-nucleotide repeats while $20 \%$ of di-nucleotide repeats and both of the two hexa-nucleotide repeats were variable. On the other hand, zero of the tri-, tetra- and penta-nucleotide repeats showed size variation between the two species (Fig. 6). The length of variable versus non-variable chloroplast microsatellites was similar but with a greater length variation for variable microsatellites in both F. crenata and F. engleriana (Fig. 7). 


\section{CONCLUSION}

Overall, the chloroplast genome of $F$. crenata will provide a useful genetic resource for future genetic studies into the foundation temperate tree genus Fagus. Specifically, the chloroplast genomes of both informal subgenera will provide useful references and sources of molecular markers to investigate phylogeographic patterns of the chloroplast within and between Fagus species. Some major questions are yet to be resolved in Fagus, including resolving taxonomic boundaries of western Eurasian Fagus populations which has remained a recalcitrant problem due to low marker resolution and high within-species genetic diversity (Denk et al., 2002) and the non-monophyly of the chloroplast of East Asian species as suggested by Sanger sequence-based data (Manos \& Stanford, 2001; Okaura \& Harada, 2002).

\section{ACKNOWLEDGEMENTS}

We would like to thank fellow lab members for their advice on this study and H. Kanehara for her assistance in the lab.

\section{ADDITIONAL INFORMATION AND DECLARATIONS}

\section{Funding}

This work was supported by the Japanese Society for the Promotion of Science Grant-in-Aid for Young Scientists A (Grant number 16748931); and a Forestry and Forest Products Research Institute grant (Grant number 201430). The funders had no role in study design, data collection and analysis, decision to publish, or preparation of the manuscript.

\section{Grant Disclosures}

The following grant information was disclosed by the authors:

Japanese Society for the Promotion of Science Grant-in-Aid for Young Scientists A: 16748931.

Forestry and Forest Products Research Institute grant: 201430.

\section{Competing Interests}

The authors declare that they have no competing interests.

\section{Author Contributions}

- James R. P. Worth conceived and designed the experiments, performed the experiments, analyzed the data, prepared figures and/or tables, authored or reviewed drafts of the paper, approved the final draft.

- Luxian Liu conceived and designed the experiments, analyzed the data, prepared figures and/or tables, authored or reviewed drafts of the paper, approved the final draft.

- Fu-Jin Wei analyzed the data, prepared figures and/or tables, approved the final draft.

- Nobuhiro Tomaru conceived and designed the experiments, contributed reagents/ materials/analysis tools, authored or reviewed drafts of the paper, approved the final draft. 


\section{DNA Deposition}

The following information was supplied regarding the deposition of DNA sequences:

Data are available at GenBank, accession number: MH171101.

\section{Data Availability}

The following information was supplied regarding data availability:

Data are available at the BioProject database:

BioProject ID: PRJNA528838.

\section{Supplemental Information}

Supplemental information for this article can be found online at http://dx.doi.org/10.7717/ peerj.7026\#supplemental-information.

\section{REFERENCES}

Castresana J. 2000. Selection of conserved blocks from multiple alignments for their use in phylogenetic analysis. Molecular Biology and Evolution 17(4):540-552 DOI 10.1093/oxfordjournals.molbev.a026334.

Csilléry K, Lalagüe H, Vendramin GG, González-Martínez SC, Fady B, Oddou-Muratorio S. 2014. Detecting short spatial scale local adaptation and epistatic selection in climate-related candidate genes in European beech (Fagus sylvatica) populations. Molecular Ecology 23(19):4696-4708 DOI 10.1111/mec.12902.

Darling ACE, Mau B, Blattner FR, Perna NT. 2004. Mauve: multiple alignment of conserved genomic sequence with rearrangements. Genome Research 14(7):1394-1403 DOI 10.1101/gr.2289704.

Darriba D, Taboada GL, Doallo R, Posada D. 2012. jModelTest2: more models, new heuristics \& parallel computing. Nature Methods 9(8):772 DOI 10.1038/nmeth.2109.

Denk T, Grimm GW, Hemleben V. 2005. Patterns of molecular and morphological differentiation in Fagus (Fagaceae): phylogenetic implications. American Journal of Botany 92(6):1006-1016 DOI 10.3732/ajb.92.6.1006.

Denk T, Grimm G, Stögerer K, Langer M, Hemleben V. 2002. The evolutionary history of Fagus in western Eurasia: evidence from genes, morphology \& the fossil record. Plant Systematics and Evolution 232(3-4):213-236 DOI 10.1007/s006060200044.

Dierckxsens N, Mardulyn P, Smits G. 2016. NOVOPlasty: de novo assembly of organelle genomes from whole genome data. Nucleic Acids Research 45(4):e18.

Doyle JJ. 1990. Isolation of plant DNA from fresh tissue. Focus 12:13-15.

Fujii N, Tomaru N, Okuyama K, Koike T, Mikami T, Ueda K. 2002. Chloroplast DNA phylogeography of Fagus crenata (Fagaceae) in Japan. Plant Systematics and Evolution 232(1-2):21-33 DOI 10.1007/s006060200024.

Heenan PB, Smissen RD. 2013. Revised circumscription of Nothofagus and recognition of the segregate genera Fuscospora. Lophozonia, \& Trisyngyne (Nothofagaceae). Phytotaxa 146(1):1-31.

Katoh K, Misawa K, Kuma K, Miyata T. 2002. MAFFT: a novel method for rapid multiple sequence alignment based on fast Fourier transform. Nucleic Acids Research 30(14):3059-3066 DOI 10.1093/nar/gkf436.

Kozlov A, Darriba D, Flouri T, Morel B, Stamatakis A. 2018. RAxML-NG: a fast, scalable, \& user-friendly tool for maximum likelihood phylogenetic inference. bioRxiv 447110.

Kumar S, Stecher G, Tamura K. 2016. MEGA7: molecular evolutionary genetics analysis version 7.0 for bigger datasets. Molecular Biology and Evolution 33(7):1870-1874 DOI 10.1093/molbev/msw054. 
Librado P, Rozas J. 2009. DnaSP v5: a software for comprehensive analysis of DNA polymorphism data. Bioinformatics 25(11):1451-1452 DOI 10.1093/bioinformatics/btp187.

Lohse M, Drechsel O, Bock R. 2007. OrganellarGenomeDRAW (OGDRAW): a tool for the easy generation of high-quality custom graphical maps of plastid and mitochondrial genomes. Current Genetics 52(5-6):267-274 DOI 10.1007/s00294-007-0161-y.

Magri D, Vendramin GG, Comps B, Dupanloup I, Geburek T, Gömöry D, Latałowa M, Litt T, Paule L, Roure JM, Tantau I, van der Knaap WO, Petit RJ, de Beaulieu J-L. 2006. A new scenario for the Quaternary history of European beech populations: palaeobotanical evidence and genetic consequences. New Phytologist 171(1):199-221 DOI 10.1111/j.1469-8137.2006.01740.x.

Manos PS, Stanford AM. 2001. The historical biogeography of Fagaceae: tracking the tertiary history of temperate and subtropical forests of the northern hemisphere. International Journal of Plant Sciences 162(S6):S77-S93 DOI 10.1086/323280.

Manos PS, Steele KP. 1997. Phylogenetic analyses of "higher" Hamamelididae based on plastid sequence data. American Journal of Botany 84(10):1407-1419 DOI 10.2307/2446139.

Mayer C. 2008. Phobos, a tandem repeat search tool for complete genomes. Version 3:12. Available at http://www.ruhr-uni-bochum.delecoevo/cm/cm_phobos.htm.

Melotto-Passarin DM, Tambarussi EV, Dressano K, De Martin VF, Carrer H. 2011. Characterization of chloroplast DNA microsatellites from Saccharum spp and related species. Genetics and Molecular Research 10(3):2024-2033 DOI 10.4238/vol10-3gmr1019.

Notredame C, Higgins DG, Heringa J. 2000. T-Coffee: a novel method for fast and accurate multiple sequence alignment. Journal of Molecular Biology 302(1):205-217 DOI 10.1006/jmbi.2000.4042.

Oh S-H. 2015. Sea, wind, or bird: origin of Fagus multinervis (Fagaceae) inferred from chloroplast DNA sequences. Korean Journal of Plant Taxonomy 45(3):213-220 DOI 10.11110/kjpt.2015.45.3.213.

Okaura T, Harada K. 2002. Phylogeographical structure revealed by chloroplast DNA variation in Japanese Beech (Fagus crenata Blume). Heredity 88(4):322-329 DOI 10.1038/sj.hdy.6800048.

Renner SS, Grimm GW, Kapli P, Denk T. 2016. Species relationships and divergence times in beeches: new insights from the inclusion of 53 young and old fossils in a birth-death clock model. Philosophical Transactions of the Royal Society B: Biological Sciences 371(1699):20150135 DOI 10.1098/rstb.2015.0135.

Shen C-F. 1992. A monograph of the genus Fagus Tourn. Ex L. (Fagaceae). PhD Dissertation. City University of New York.

Tsukada M. 1982. Late-Quaternary development of the Fagus forest in the Japanese Archipelago. Japanese Journal of Ecology 32:113-118.

Vitelli M, Vessella F, Cardoni S, Pollegioni P, Denk T, Grimm GW, Simeone MC. 2017. Phylogeographic structuring of plastome diversity in Mediterranean oaks (Quercus Group Ilex, Fagaceae). Tree Genetics \& Genomes 13(1):3 DOI 10.1007/s11295-016-1086-8.

Wyman SK, Jansen RK, Boore JL. 2004. Automatic annotation of organellar genomes with DOGMA. Bioinformatics 20(17):3252-3255 DOI 10.1093/bioinformatics/bth352.

Yan M, Xiong Y, Liu R, Deng M, Song J. 2018. The application \& limitation of universal chloroplast markers in discriminating East Asian evergreen oaks. Frontiers in Plant Science 9:569 DOI 10.3389/fpls.2018.00569.

Yang Y, Zhu J, Feng L, Zhou T, Bai G, Yang J, Zhao G. 2018. Plastid genome comparative \& phylogenetic analyses of the key genera in Fagaceae: highlighting the effect of codon composition bias in phylogenetic inference. Frontiers in Plant Science 9:82 DOI 10.3389/fpls.2018.00082. 